\title{
Uma ferramenta computacional para simulação de espalhamento de fluidos baseada em autômatos celulares bidimensionais estocásticos
}

\author{
Marcos V. B. Lima ${ }^{1}$, Cíntia C. Oliveira ${ }^{1}$, Danielli A. Lima ${ }^{1}$ \\ ${ }^{1}$ Instituto Federal do Triângulo Mineiro (IFTM) - Campus Avançado Patrocínio \\ Av. Líria Terezinha Lassi Capuano no 255 - 38.740-000 - Patrocínio - MG - Brazil \\ marcoslimaptc@gmail.com, cintiaoliveira@iftm.edu.br, danielli@iftm.edu.br
}

\begin{abstract}
One of the main causes of water pollution are the mineral extraction and fossil fuels from soil or even oceans. The intense search for these natural resources for creating derivatives grown in large scale, increasing, consequently, their exploitation. However, its leak in nature can cause serious problems to the humans and animals lives. In this context, this paper presents a cellular automata study and its applications, and proposes a model which simulates a fluid slick model and then presents a prototype system to support these simulations. Experiments and tests were conducted to examine how the variables involved affect the fluid spreading in the simulated environment.
\end{abstract}

Resumo. Uma das principais causas de poluição da água está na extração de minerais e combustíveis fósseis do solo ou até mesmo de oceanos. A busca intensa por esses recursos naturais para criação de produtos derivados cresce em larga escala, aumentando, consequentemente, a sua exploração. Entretanto, o seu vazamento na natureza pode causar sérios problemas à vida dos humanos e animais. Neste contexto, o presente trabalho apresenta um estudo dos autômatos celulares e suas aplicações, e propõe um modelo para realizar simulações de espalhamento de fluidos, e posteriormente apresenta um protótipo do sistema para suportar essas simulações. Experimentos e testes foram realizados para analisar como as variáveis envolvidas afetam o espalhamento de fluidos dentro do ambiente simulado.

\section{Introdução}

Estudos relacionados ao espalhamento de fluidos auxiliam principalmente as indústrias a quantificarem os níveis de espalhamento de fluidos tóxicos sobre uma determinada região, bem como o estudo de técnicas de remediação [Lehikoinen et al. 2015]. No caso do petróleo ou de outro rejeito tóxico, o espalhamento pode ocorrer através da elevação natural ou artificial devido à perfuração de poços artesianos [Mondol et al. 2015]. Assim, a excessiva extração desses materiais causam sérios prejuízos naturais na presença de um acidente, afetando, principalmente, a qualidade da água, causando a degradação do solo, prejudicando a vida dos animais.

O objetivo de um modelo para a previsão do espalhamento de fluidos é a determinação da evolução da poluição de um rejeito ou resíduo frente ao tempo em um ambiente simulado. Direção do vento ou correnteza, direção e velocidade das ondas são fatores determinantes num vazamento de grande porte. Esses modelos matemáticos 
e computacionais podem ser parte de um sistema real que combinam um sistema de informação geográfica (GIS) e imagens de satélite com um modelo de espalhamento de fluidos [Mohamadi et al. 2015]. Entretanto, esta abordagem envolvendo processamento de imagens de satélite não será investigada no presente trabalho.

Uma ferramenta útil na elaboração de simulações gerenciadas por computador são estabelecidas por modelos matemáticos conhecidos como Autômatos Celulares (ACs). Existem diversas aplicações no uso de ACs, dentre elas, podemos citar a modelagem de fenômenos naturais [Duff et al. 2015], ambientais [Lima and Lima 2014], físicos [Jian-hua et al. 2015], biológicos [Wolfram 2002] ou sociais [Castro and Lima 2013], que seriam muito difíceis de serem modeladas pelas equações diferenciais, sendo estas as mais utilizadas nesse tipo de tarefa. Além disso, os ACs possuem alto nível de paralelismo, quando implementados em um hardware paralelo.

O presente trabalho tem como objetivo: (i) introduzir o conceito de autômatos celulares e apresentar suas características, (ii) implementar o método de simulação do espalhamento de fluidos baseado em autômatos celulares bidimensionais a partir de adaptações de modelos precursores [Ha et al. 2012, Karafyllidis 1997, Dilão 1993]. A fim de avaliar o modelo sugerido, (iii) testes em diferentes situações foram realizados. As simulações fornecem o tempo de espalhamento total e a partir de várias simulações é possível obter a média geral do tempo de espalhamento de resíduos no ambiente aquático. Além disso, um protótipo do sistema como um todo foi elaborado e novos parâmetros vêm sendo adicionados para aumentar o realismo e a precisão do modelo apresentado neste trabalho.

\section{Fundamentação teórica e trabalhos relacionados}

\subsection{Autômatos celulares}

Um AC é composto por um reticulado com uma dimensão $d$ dividido em células ou unidades processadoras, sendo que, cada célula $C$ é representada por um estado. As células modificam seus estados a cada passo de iteração de acordo com uma regra de transição. Podemos aplicar a regra de transição por $T$ passos de tempo para obter a evolução espaço-temporal do reticulado do AC. A regra estabelecida por uma função de transição indica o novo símbolo a ser escrito na célula do reticulado de acordo com seu estado atual e dos estados de suas vizinhas (regra local). Em sua definição mais usual, a atualização dos estados se dá de forma síncrona e utiliza uma regra determinística, isto é, a cada passo de tempo todas as $N$ células do reticulado são atualizadas. No modelo proposto neste trabalho, o sistema de regras é probabilístico, ou seja, é possível alterar o estado de uma célula a partir de uma função de probabilidade, isso significa que as regras de atualização são probabilísticas e, portanto, o estado de uma célula em um determinado instante depende probabilisticamente dos estados de suas células vizinhas no passo de tempo imediatamente anterior [Lima and Lima 2014].

A estrutura dos ACs unidimensionais (1D) é a mais estudada. Para um AC com regra de atualização determinística, a mudança de estado de uma célula depende de $m$ vizinhas expressas por $m=(2 r+1)$, sendo $r$ o raio do AC [Oliveira 2003]. Para ilustrar um AC unidimensional com regra de atualização determinista, considere a Figura 1 (a), que aborda uma modelagem conhecida como regra 30 [Wolfram 2002], contendo um reticulado de 6 células sendo que o estado inicial de cada célula é apresentado em $t=0$. Uma regra binária de raio 1 é aplicada e a vizinhança de cada célula é formada 
por três elementos: a própria célula e suas duas vizinhas adjacentes (à esquerda e à direita). Como esse AC é binário (2 estados possíveis), existem 8 diferentes vizinhanças, da 000 a 111. A regra em si é dada pelos 8 bits de saída associados a cada vizinhança possível: 01111000. Na Figura 1 (b) vemos a atualização do reticulado de $N=6$ por 2 passos de tempo a partir de sua configuração inicial $101110 \mathrm{em} t=0$. A cada passo, cada célula do reticulado é atualizada identificando-se sua vizinhança e seu novo estado é dado pelo bit de saída correspondente na regra de transição. Observe como exemplo, a célula de símbolo 1 , destacada em $t=0$, seu próximo estado será 0 em $t=1$. O reticulado é submetido a condições periódicas de contorno, sendo que a primeira célula é vizinha imediata da última, e vice-versa. Aplicando-se esse procedimento para todas as células do reticulado de forma síncrona, tem-se a nova configuração do reticulado a cada passo de tempo [Castro and Lima 2013].

Os ACs bidimensionais (2D) são amplamente conhecidos na literatura

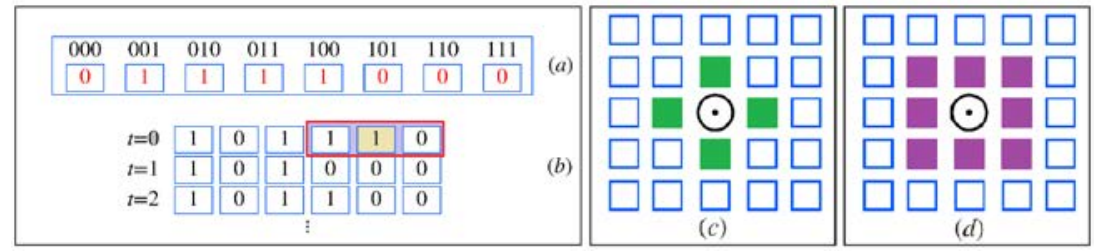

Figura 1. (a) Regra de transição de raio 1. (b) Evolução do AC por $T=2$ passos de tempo. (c) Vizinhança de Von Neumann. (d) Vizinhança de Moore [Lima and Lima 2014].

[Oliveira 2003]. Essa dimensão contempla variadas formas para a consideração da vizinhança de uma célula, sendo que as duas principais estão apresentadas na Figura 1 (c) e (d), tal que o símbolo $\odot$ representa a célula central e o símbolo $\square$ identifica a célula vizinha. Dentre as aplicações de ACs 2D, o modelo mais conhecido é o jogo matemático proposto por Conway em 1970 denominado Game of Life apresentado na Figura 2.

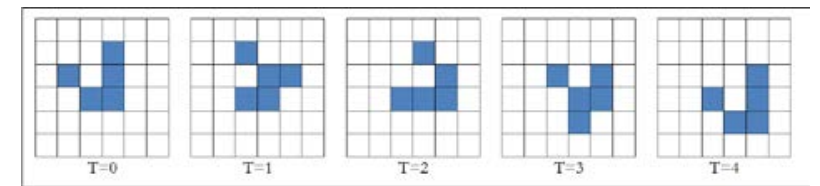

Figura 2. Evolução do AC "Game of Life" por $T=4$ passos de tempo.

\subsection{Modelos de espalhamento de fluidos baseados em Autômatos Celulares}

Um dos trabalhos conhecidos nessa temática foi o estudo para modelagem de derramamento de fluidos através de um modelo microscópico proposto por [Dilão 1993]. Neste trabalho, o autor definiu o comportamento de espalhamento de fluidos, baseando-se no estudo de caso de derramamento no estuário Tejo. Para isso, o autor construiu um programa dedicado a partir de fotos capturadas por satélite. Para alcançar o realismo necessário, regras baseadas em Autômatos Celulares e Lattice Gas foram utilizadas num cenário microscópico. O AC apresentou um reticulado bidimensional com células no formato triangular e as partículas deslocam-se no decorrer das ligações entre essas células por um período de tempo constante. As colisões entre essas partículas foram resolvidas de 
maneira não-determinística.

Um modelo para a predição de derramamento de óleo e sua movimentação e espalhamento foi proposto em [Karafyllidis 1997], utilizando-se Autômatos Celulares. O modelo proposto neste trabalho foi capaz de prever derramamento de óleo em regiões com limites complexos. Os efeitos de vento, correnteza e evaporação do óleo foram considerados. Um algoritmo para a simulação de derramamento de óleo, bem como regiões geográficas hipotéticas foi considerado. Os resultados da simulação ficaram em conformidade com o real movimento e espalhamento de derramamento de óleo.

No trabalho de [Ha et al. 2012] um modelo macroscópico foi proposto para analisar o fenômeno de espalhamento de óleos em oceanos quando o mesmo foi poluído por um navio de extração. O espaço ocupado pelo oceano foi convertido em células, que continham informações, tais como, quantidade de óleo, temperatura do oceano e a direção do vento. Dois estados denominados: limpo e poluído são definidos por cada célula. $O$ óleo espalha nas células vizinhas através de regras de atualização. No trabalho foram empregadas 3 regras de atualização. A primeira considera que o óleo apenas espalha para uma célula $x_{i}$ a partir da vizinhança dessa célula, se existe uma célula vizinha $x_{j}$ com mais óleo que a célula $x_{i}$. A segunda considera que o óleo evapora numa proporção de acordo com a temperatura do oceano a cada passo de tempo. A terceira considera que a propriedade do óleo é afetada pela direção e velocidade do vento. A simulação mostrou que o modelo de cada célula é definido pela combinação de eventos discretos e que o tempo discreto da simulação foi capaz de avaliar um ambiente com $300 \times 300$ células com $10^{3} \mathrm{kl}$ em uma determinada corrente de vento.

Mais tarde, no trabalho de [Vourkas and Sirakoulis 2012], uma modelagem mais complexa sobre a utilização dos ACs, que são inerentemente paralelos, foram aplicadas com êxito para a modelagem do derramamento de resíduos tóxicos. Nesse trabalho, os autores definem a área do espalhamento como uma matriz de células de quadrados idênticos, e o tamanho das células do AC é dado entre a precisão de cálculo e os recursos computacionais necessários, para realização dos cálculos em tempo real. Além disso, uma implementação do modelo de autômatos celulares (ACs) foi apresentada na plataforma FPGA (Field Programmable Gate Arrays). Foram avaliadas as semelhanças entre os ACs e a plataforma FPGA. Posteriormente, foram discutidos os principais desafios da implementação, bem como o mapeamento apropriado e favorável do hardware para a exploração das características do ACs. Adicionalmente, foi realizada uma significativa avaliação de desempenho em comparação com as soluções de software e hardware correspondentes.

\section{Modelo proposto}

O modelo proposto é fortemente baseado nos modelos de [Dilão 1993], [Karafyllidis 1997] e [Ha et al. 2012]. Um modelo inicial básico foi criado, posteriormente mais parâmetros foram sendo agregados ao modelo a fim de proporcionar realismo às simulações realizadas com intuito experimental. As simulações apresentadas neste trabalho têm o intuito de mostrarem uma visualização sob o ponto de vista macroscópico. A seguir, o modelo será apresentado, e será detalhada cada modificação que foi realizada em relação aos modelos precursores.

No modelo aqui proposto considera-se uma rede bidimensional com simetria quadrada, vizinhança de Moore e cada célula da malha representa o estado de uma área/região do ambiente. No trabalho de [Ha et al. 2012] consideram-se dois estados 
possíveis para uma célula: o estado associado à cor azul representa uma área de água intacta, à cor preta uma área que foi fortemente contaminada. Entretanto, no modelo aqui apresentado outros estados foram acrescidos. Esta modificação foi inspirada no modelo de [Hernandez et al. 2007] que também subdividiu o problema em outros estados a fim de dar mais realismo à simulação. A Figura 3 (a) e (b) apresenta a nova divisão do estado de poluição no modelo precursor de [Ha et al. 2012].

A regra de atualização proposta neste trabalho é probabilística e periódica e os estados da malha são atualizados a cada iteração de forma síncrona, sendo que a mesma é baseada do modelo de [Ha et al. 2012]. A ideia geral da regra de atualização será descrita a seguir. Se num certo instante uma célula está no estado azul (porção de água não contaminada) e uma das células vizinhas está no estado de contaminação, existe uma probabilidade não nula de, no instante seguinte, a célula mudar para o estado preto (Figura 3 (b) cor 1 ). A cada $t=2$ o estado da célula é alterado incrementando-se a cor (ver Figura 3 (b) cor 2 até cor 4). Ou seja, quando uma célula está no estado de contaminação, ela vai manter-se nesse estado durante um tempo finito (neste caso, $t=12$ ). Se uma célula da malha está no estado azul claro (porção de água levemente contaminada), então ficará nesse estado indefinidamente $(t=\infty)$ ou até que sofra um processo de evaporação definido por uma constante de probabilidade de evaporação $\beta$. Ou seja, é possível que a partir de uma quantidade de evaporação (que varia de acordo com o fluido estudado), a porção contaminada volte para o estado intacto novamente. Uma vez que cada célula tem uma probabilidade $p_{i j}=\beta$ de evaporar. Ao evaporar, essa célula diminui a concentração da contaminação, ou seja, vai para o próximo estado da Figura 3. Se existir vento ou direção de correnteza numa certa direção, a probabilidade de transição azul $\rightarrow$ para o estado de poluição é maior nessa direção. Assim, define-se o caráter não-determinista na evolução do autômato celular, similar ao que já foi abordado em [Dilão 1993]. No modelo aqui apresentado, a partir do momento que a célula entra no estado de contaminação, cada estado muda de acordo com o tempo para acontecer o espalhamento $(t=2)$, enquanto que no modelo de [Hernandez et al. 2007] essa mudança era dada por uma regra estocástica. Uma primeira adaptação que considera que uma célula $(i, j)$ tem a probabilidade $p_{i j}=x$ de se contaminar, sendo que $x \in[0,1]$. Ou seja, basta uma célula ter uma vizinha no estado contaminado que ela tem uma probabilidade não nula de no próximo instante de tempo passar do estado intacto para o estado contaminado, diferentemente do modelo de [Ha et al. 2012], onde a dispersão era realizada a partir de diferenças de quantidades entre células vizinhas.

Em relação ao fator vento abordado em [Karafyllidis 1997] regras estatísticas foram usadas. Neste trabalho, não será utilizado o parâmetro vento. Um parâmetro bem similar será usado que é a direção e velocidade da correnteza, para essa modelagem uma matriz de preferências de dimensão $m \times m$ foi usada. Essa matriz representa a vizinhança de Moore, adotada em modelos de ACs para dinâmica de partículas. De acordo com [Pereira et al. 2011], essa matriz indica uma maior preferência da partícula para uma determinada direção, mostrando-se muito eficaz para modelar o fator velocidade e direção da correnteza. Essa matriz poderá ser adaptada para modelar outros parâmetros do sistema, tais como, vento e densidade. A proposta considera uma matriz de preferências, tal que essa dimensão representa a vizinhança do $\mathrm{AC}$ com raio $r=1$. Neste caso, a matriz de preferências indica que a velocidade da correnteza é de $v_{\max }=1$. Ou seja, $v_{\text {max }}=\sum_{i=1}^{m} x_{i j}$ é a soma dos pesos das células pertencentes à vizinhança $m\left(x_{i j} \in m\right)$. 
A matriz de preferências utilizada no modelo pode ser vista na Figura 3(c). Caso seja necessário alterar a direção do vento, basta rotacionar a matriz para a direção desejada, conforme proposto nos algoritmos de dinâmica de partículas [Pereira et al. 2011]. A probabilidade de infecção de uma área intacta é dada por $p_{i j}=\sum_{i=1}^{m} \sum_{j=1}^{m} m_{a} t_{i j} \times \beta$, sendo que mat $_{i j}$ é a matriz de preferências e $\beta$ é a constante de probabilidade de evaporação.

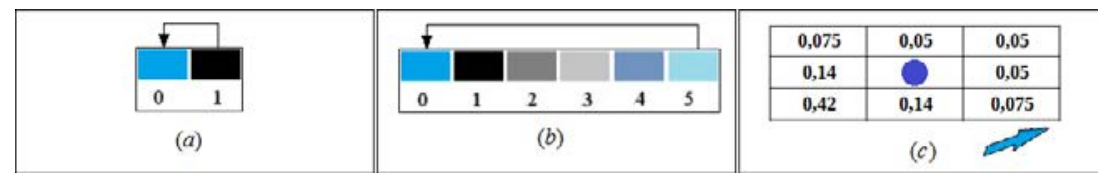

Figura 3. Cores correspondes aos estados das células: (a) modelo proposto por $\mathrm{Ha}$ (2012) adaptado para este problema. (b) modelo proposto neste trabalho. (c) Matriz de preferências para raio 1. A seta azul indica a direção da correnteza para a matriz de preferência e o círculo azul representa uma região intacta.

\section{Resultados experimentais}

Os testes apresentados nesta seção foram elaborados utilizando-se a linguagem de programação $\mathrm{C}++\mathrm{e}$ foram divididos em duas partes: a primeira trata os testes preliminares para validação do comportamento da regra de atualização e a segunda os testes de análise temporal, que analisa estatisticamente o tempo gasto para que a espalhamento se propagasse por todo o reticulado. Cada experimento avalia cada um dos parâmetros possíveis do modelo proposto neste trabalho.

\subsection{Análise visual}

Inicialmente foram realizados alguns testes para a verificação da formação de padrões na evolução do espalhamento de fluidos sem a utilização da matriz de preferências e foram contrastados com o modelo de [Ha et al. 2012]. Os experimentos representam um cenário onde um reticulado de dimensões $80 \times 80$ foi considerado. Além disso, um foco de contaminação inicial é colocado na célula central do reticulado no instante $t=0$.

Primeiramente, uma simulação no tempo $t=5$ é apresentada na Figura 4 (a) e mostra o espalhamento ainda em condições preliminares à sua difusão ao longo do reticulado. Na Figura 4 (b) tem-se a simulação do modelo de [Ha et al. 2012] em $t=20$ sem a constante de evaporação $\beta$, e na Figura 4 (c) tem-se a simulação do mesmo modelo também em $t=20$, mas considerando-se $\beta=0.05$. Nesta primeira análise, a probabilidade de contaminação $\left(p_{i j}=0.7\right)$ é a mesma, quando tem-se uma ou mais células vizinhas contaminadas. Também é possível observar que com o passar do tempo, tem-se que ao centro, a tonalidade da água é reestabelecida e a poluição é espalhada radialmente, já que o modelo é o sem vento. Na Figura 4 (d) tem-se a alteração do aumento de estados da poluição em relação ao modelo precursor no instante de tempo $t=20$, considerando-se $\beta=0.05$ e $p_{i j}=0.7$. Neste caso, o estado contaminado apresentou cinco subestados, sendo que a alteração para o estado seguinte ocorre a cada $t=2$ passos de tempo. Conforme é observado, existe um atraso na recuperação da tonalidade da água, que é ocasionado devido ao tempo do estado "contaminado" em sua totalidade ser maior $(t=12)$ em relação ao modelo-base para a construção deste trabalho [Ha et al. 2012], que utilizou apenas dois estados. A Figura 4 (e) representa a simulação no instante $t=35$, e a formação radial do espalhamento já encontra-se mais acelerada. Esse fato é ocasionado devido à constante 
de evaporação $\beta=0.05$ ser muito pequena. Além disso, uma formação radial pode ser observada em relação à proporção e formato da área contaminada, o que é garantido pela simulação do modelo com ausência de vento ou correnteza.

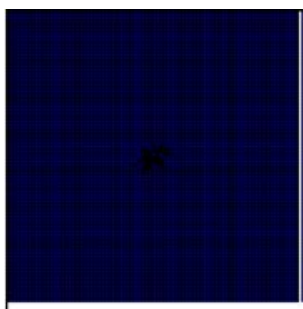

(a)

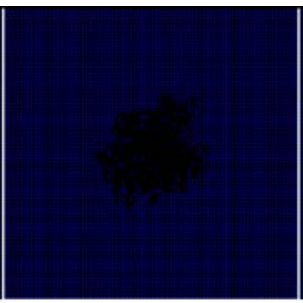

(b)

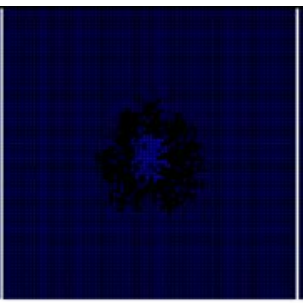

(c)

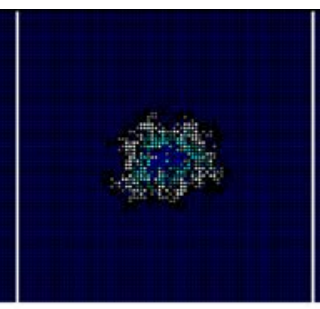

(d)

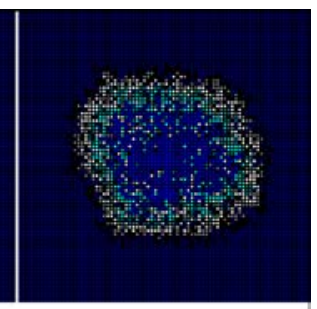

(e)

Figura 4. Observações da simulação do modelo baseado em ACs para o espalhamento de fluidos. (a) Configuração no instante $t=5$. Configuração no instante $t=20$ : (b) Modelo baseado em [Ha et al. 2012] sem evaporação, (c) Modelo baseado em [Ha et al. 2012] com evaporação e (d) Modelo aqui proposto. (e) Configuração no instante $t=35$ do modelo aqui proposto.

\subsection{Análise estatística do tempo de propagação}

Para validar o modelo sob o ponto de vista matemático, foram realizadas simulações com presença de correnteza, utilizando-se a matriz de preferências apresentada na seção anterior. Os experimentos avaliam reticulados de diferentes dimensões e formatos. Cada um desses reticulados apresenta um único foco de contaminação inicial $(t=0)$, na célula de posição central do reticulado. Foram realizadas 100 simulações para cada um dos parâmetros propostos e observou-se os tempos $(T)$ de propagação gastos para que toda a área do reticulado fosse contaminada. Portanto, para que o critério de parada fosse atingido, a constante de evaporação $\beta$ não foi utilizada. Histogramas de classe foram plotados para a validação dos tempos $T$ de cada um dos modelos (Figura 5).

Os histogramas das Figuras 5 (a) e (b) referem-se ao experimentos com reticulados quadrados de dimensão $50 \times 50$ e $80 \times 80$. Para o experimento referente ao histograma da Figura 5 (a), tem-se média $\bar{x}=53.19$, mediana $\tilde{x}=53.17$ e moda $m o=52.43$. Para o experimento referente ao histograma da Figura 5 (b) os valores médios são maiores devido ao tamanho do reticulado que impacta diretamente neste resultado. Neste caso, tem-se média $\bar{x}=69.36$, mediana $\tilde{x}=68,83$ e moda $m o=69.08$. Nestes dois primeiros gráficos é possível perceber que a distribuição dos dados referentes às simulações apresentam uma melhor distribuição, o que torna essa configuração de ambiente mais significativa.

Os histogramas das Figuras 5 (c) e (d) referem-se ao experimentos com reticulados não quadrados de dimensão $30 \times 50$ e $50 \times 80$, respectivamente. Para o experimento referente ao histograma da Figura 5 (c), tem-se média $\bar{x}=40.17$, mediana $\tilde{x}=39.89$ e moda $m o=39.02$. Para o experimento referente ao histograma da Figura 5 (d), os valores médios são maiores, devido ao tamanho do reticulado que impacta diretamente neste resultado. Neste caso, tem-se média $\bar{x}=48.28$, mediana $\tilde{x}=47.45 \mathrm{e}$ moda $m o=46.94$. Em relação aos experimentos com reticulados quadrados, é possível observar que a distribuição naqueles é mais próximo à distribuição simétrica, quando comparados com os experimentos de reticulados não quadrados. Como é possível observar, os resultados referentes aos experimentos com reticulados não quadrados apresentam distribuições assimétricas positivas. As distribuições assimétricas são características de 
resultados onde $\bar{x}>\tilde{x}>m o$. Além disso, os valores anotados de cada simulação são menores em relação aos dos experimentos anteriores, isso deve-se principalmente ao fato da dimensão do reticulado e formato. Reticulados com formato quadrado proporcionam maior velocidade ao espalhamento. Uma vez que, o reticulado utilizado neste trabalho não possui contorno periódico. Essa ausência de periodicidade no reticulado estimula a criação de barreiras por ausência de vizinhança para a aplicação da regra de transição, sendo esta o motor de toda a simulação.

Pode-se observar que a partir destes gráficos as distribuições das adaptações reali-

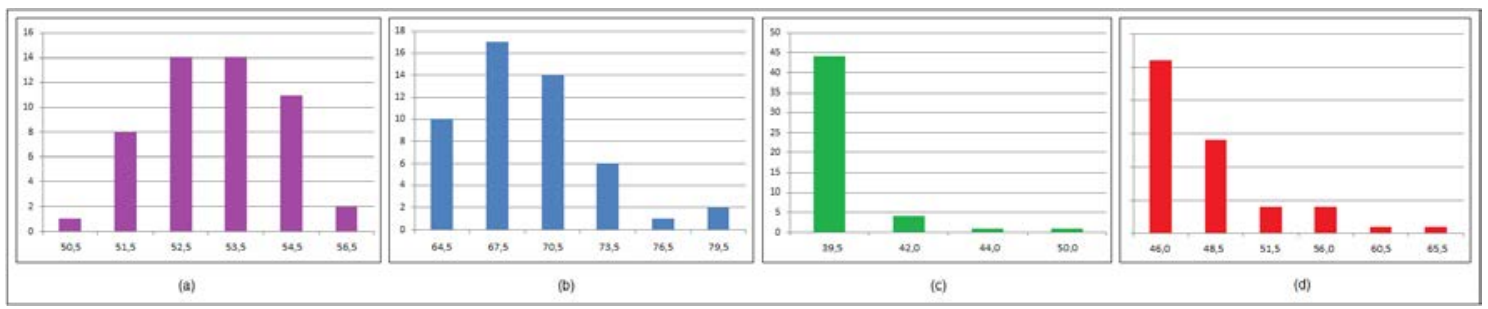

Figura 5. (a) Histograma de classes para os reticulados de dimensões (a) $50 \times$ 50. (b) $80 \times 80$. (c) $30 \times 50$. (d) $50 \times 80$.

zadas para reticulados quadrados possuem maior confiança em relação aos experimentos realizados com reticulados não quadrados. Uma atenção especial para os gráficos de experimentos realizados com reticulados não quadrados é que estes apresentam mais valores que estão distantes da média. Esse resultado mostra que determinadas configurações apresentam mais dificuldade para que o ambiente fosse totalmente coberto pela contaminação.

\subsection{Protótipo do sistema de simulação de espalhamento de fluidos}

Esta seção apresenta o conjunto de interfaces propostas neste trabalho para a criação do sistema de simulação de espalhamento de fluidos, bem como a breve descrição de suas funcionalidades. A ferramenta inicialmente foi desenvolvida em linguagem C++ e será importada para as interfaces do Java juntamente com as opções de configuração do espalhamento. Na Figura 6 é possível observar a tela proposta para o sistema de simulação com as opções de configuração do espalhamento. No canto superior esquerdo, temos a representação do ambiente aquático ainda intacto (antes do espalhamento começar). No canto superior direito, um painel para escolha do tipo de fluido, bem como o símbolo de representação do mesmo. Cada fluido possui por padrão valores automáticos relativos à sua densidade, viscosidade, massa e peso, com exceção da opção "Outro" que permite ao usuário alterar o nome e os parâmetros de um novo fluido. Na parte inferior da tela, encontramos as demais opções de configuração do espalhamento, onde são reguladas as dimensões do ambiente, o tempo inicial e final do espalhamento $(T)$, e a intensidade de correnteza e direção do vento. A opção "Estatísticas" exibe a tela apresentada à direita e nela valores dos dados inseridos são mostrados, tais como média, moda e mediana, assim como outros valores que serão agregados posteriormente.

\section{Conclusões e trabalhos futuros}

Neste trabalho foi investigado um modelo de autômatos celulares bidimensionais estocásticos fundamentado em modelos precursores aplicados à simulação de espalhamento de fluidos. Para a modelagem do AC detalhado no trabalho, considerou-se 


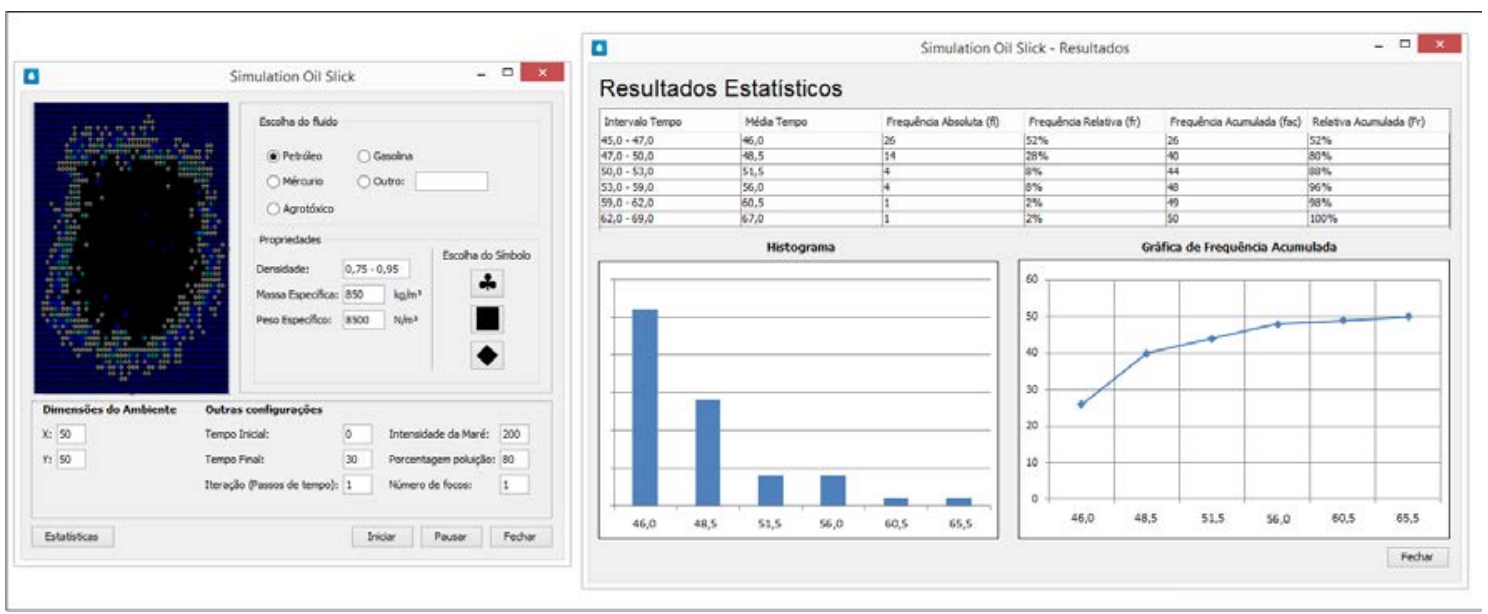

Figura 6. Modelo de tela proposto para o ambiente da simulação em funcionamento e modelo de tela proposto para exibir os resultados estatísticos obtido pelas simulações.

a implementação descrita por [Ha et al. 2012], [Dilão 1993] e as complementações idealizadas por [Karafyllidis 1997]. Todas as adaptações lógicas foram concebidas com a análise desses modelos precursores. Além disso, uma análise das características do modelo desenvolvido foi abordada.

Com a execução do modelo descrito foi possível gerar dados visuais, onde os comportamentos padrões de espalhamento de fluidos puderam ser observados. Além disso, dados estatísticos foram obtidos relacionando-se a média das iterações com as adaptações de cada um dos parâmetros de dimensão e formato do reticulado. A grande vantagem de se usar o modelo aqui desenvolvido, reside no fato de que o mesmo é flexível para a entrada dos parâmetros já adotados e de novos que poderão ser inseridos. Entretanto, não é possível garantir que apenas os comportamentos inseridos por si o já comprovam o com exatidão a área contaminada a partir das simulações do modelo. Mas, elas iniciam uma análise que pode ser aprofundada em um trabalho de pesquisa posterior.

Como continuidade desse trabalho, seria interessante a adaptação do modelo 2D para um modelo 3D, considerando-se neste caso profundidade de contaminação, tornando a modelagem mais realista e precisa. Uma adaptação para o espalhamento de outros tipos de fluidos em diferentes meios também poderia ser incorporada ao trabalho. Para alcançar o paralelismo e simulações mais rápidas do modelo poderia ser utilizada uma implementação em hardware paralelo, uma vez que toda a motivação para o emprego de ACs na simulação reside na possibilidade de uma implementação eficiente, similar ao trabalho de [Vourkas and Sirakoulis 2012]. Além disso, um estudo de caso considerandose um espalhamento de fluidos real poderia ser realizado, a exemplo, o espalhamento de rejeitos tóxicos na cidade de Mariana-MG em 2015.

\section{Referências}

Castro, A. P. and Lima, D. A. (2013). Autômatos celulares aplicados a modelagem de dinâmica populacional em situação de risco. In 4th Workshop of Applied Computing for the Management of the Environment and Natural Resources WCAMA 2013 CSBC, Universidade Federal de Alagoas. 
Dilão, R. (1993). Autômatos celulares, máquinas de turing ou a natureza como máquina de cálculo. Colóquio Ciências, 12:3-20.

Duff, T. J., Chong, D. M., and Tolhurst, K. G. (2015). Using discrete event simulation cellular automata models to determine multi-mode travel times and routes of terrestrial suppression resources to wildland fires. European Journal of Operational Research, 241(3):763-770.

Ha, S., Cha, J.-H., Ku, N.-K., and Lee, K.-Y. (2012). Cell-based discrete event and discrete time simulation for the prediction of oil slick movement and spreading in ocean environment. Transactions of the Society of CAD/CAM Engineers, 17(1):45-53.

Hernandez, L., Hoya, S., Martan, A., and Rodriguez, G. (2007). Modelling forest fire spread using hexagonal cellular automata. Applied Mathematical Modelling, 31(6):1213 - 1227.

Jian-hua, Z., Tao, J., Sheng-an, W., and Jia-wei, M. (2015). Research of cellular automata traffic flow model for variable traffic flow density. Traffic, 10(20):26-25.

Karafyllidis, I. (1997). A model for the prediction of oil slick movement and spreading using cellular automata. Environment international, 23(6):839-850.

Lehikoinen, A., Hanninen, M., Storgard, J., Luoma, E., Mantyniemi, S., and Kuikka, S. (2015). A bayesian network for assessing the collision induced risk of an oil accident in the gulf of finland. Environmental science and technology, 49(9):5301-5309.

Lima, H. A. and Lima, D. A. (2014). Autômatos celulares estocásticos bidimensionais aplicados à simulação de propagação de incêndios em florestas homogêneas. In 5th Workshop of Applied Computing for the Management of the Environment and Natural Resources WCAMA 2014 CSBC, Universidade de Brasilia.

Mohamadi, B., Xie, Z., and Liu, F. (2015). Gis based oil spill risk assessment model for the niger deltas vegetation. Nature Environment and Pollution Technology, 14(3):545.

Mondol, M. R., Keshavmurthy, S., Lee, H.-J., Hong, H.-K., Park, H.-S., Park, S.-R., Kang, C.-K., and Choi, K.-S. (2015). Recovery of wild pacific oyster, crassostrea gigas in terms of reproduction and gametogenesis two-years after the hebei spirit oil spill accident off the west coast of korea. Continental Shelf Research, 111:333-341.

Oliveira, G. M. B. (2003). Automatos celulares: aspectos dinâmicos e computacionais. III Jornada de Mini-cursos em Inteligência Artificial (MCIA) - Sociedade Brasileira de Computação, 8:297 - 345.

Pereira, L. A., Duczmal, L. H., and Cruz, F. R. (2011). Simulação de evacuação emergencial via autômatos celulares: Uma proposta de modificação do modelo de schadschneider. In XXXII Congresso Nacional de Matemática Aplicada e Computacional, pages 692-698.

Vourkas, I. and Sirakoulis, G. C. (2012). Fpga based cellular automata for environmental modeling. In Electronics, Circuits and Systems (ICECS), 2012 19th IEEE International Conference on, pages 93-96. IEEE.

Wolfram, S. (2002). A New Kind of Science. Wolfram Media - (1st edition): 1197 2006-09-19T07:35:05.000+0200. 